\title{
In-hospital death in acute coronary syndrome was related to admission glucose in men but not in women
}

Julio Yoshio Takada*, Rogério Bicudo Ramos, Larissa Cardoso Roza, Solange Desiree Avakian, José Antonio Franchini Ramires and Antonio de Pádua Mansur

\begin{abstract}
Background: Admission hyperglycaemia is associated with mortality in patients with acute coronary syndrome (ACS), but controversy exists whether hyperglycaemia uniformly affects both genders. We evaluated coronary risk factors, gender, hyperglycaemia and their effect on hospital mortality.

Methods: 959 ACS patients (363 women and 596 men) were grouped based on glycaemia $\geq$ or $<200 \mathrm{mg} / \mathrm{dL}$ and gender: men with glucose $<200 \mathrm{mg} / \mathrm{dL}$ (menG-); women with glucose $<200 \mathrm{mg} / \mathrm{dL}$ (womenG-); men with glucose $\geq 200 \mathrm{mg} / \mathrm{dL}$ (menG+); and women with glucose $\geq 200 \mathrm{mg} / \mathrm{dL}$ (womenG+). A logistic regression analysis compared the relation between gender and glycaemia groups and death, adjusted for coronary risk factors and laboratory data.

Results group: menG- had lower mortality than menG + (OR=0.172, IC95\% 0.062-0.478), and womenG + (OR=0.275, IC95\% 0.090-0.841); womenG- mortality was lower than menG $+(\mathrm{OR}=0.230, \mathrm{IC} 95 \%$ 0.074-0.717). No difference was found between menG + vs womenG $+(p=0.461)$, or womenG- vs womenG $+(p=0.110)$. Age $(O R=1.067$, IC95\% 1.031-1.104), EF (OR=0.942, IC95\% 0.915-0.968), and serum creatinine $(O R=1.329, I C 95 \% 1.128-1.566)$ were other independent factors related to in-hospital death.

Conclusions: Death was greater in hyperglycemic men compared to lower blood glucose men and women groups, but there was no differences between women groups in respect to glycaemia after adjustment for coronary risk factors.
\end{abstract}

Keywords: Mortality, Myocardial infarction, Hyperglycaemia, Sex, Glycaemia, In-hospital prognosis

\section{Introduction}

Acute coronary syndromes (ACS) are a set of presentations of coronary artery disease (CAD) and have in common unstable atherosclerotic coronary plaque. Unstable angina (UA), non-ST-elevation myocardial infarction (NSTEMI), and ST-elevation myocardial infarction (STEMI) have differences in treatments and outcomes, but some markers of poor prognosis are common, such as clinical risk scores or several laboratory factors, including C-reactive protein, hyperglycaemia, and elevated brain natriuretic peptide type $B[1,2]$.

\footnotetext{
*Correspondence: julio.takada@incor.usp.br

Heart Institute (InCor), University of São Paulo Medical School, Avenue Enéas de Carvalho de Aguiar, 44, 05403-000 São Paulo, Brazil
}

Admission blood glucose (ABG) is a risk factor of worse in-hospital and long-term prognosis in ACS [3,4], both in diabetic and nondiabetic patients [5], but its influence on mortality can be different along time after an acute event [6,7], and according to the diagnosis of patients admitted to intensive care units [8]. In ACS patients, women have more risk factors for $C A D$ and are older than men, so after adjustment for confounders, this difference disappears, with the exception of STEMI patients when women still have higher mortality than men [9-11]. Champney et al [12] reported that at ages 50 to 70 years, women with STEMI do have an increased adjusted mortality risk in comparison with men, but not in other age ranges. On the other hand, different from that observed in men, ABG had a non-uniform relationship 
with ACS mortality in women, as described by Cubbon et al [13], who found a proportional increase in the hazard ratio for mortality based on the interaction between sex and ABG with an increase in glucose levels in men. Women however did not have a greater risk of mortality at levels of glucose $>11.1 \mathrm{mmol} / \mathrm{L}(200 \mathrm{mg} / \mathrm{dL})$.

This study evaluated the influence of ABG on hospital mortality in ACS and its subgroups of presentations, in particular whether this influence occurs in women in the same way that it occurs in men.

\section{Methods}

\section{Study design and population}

Patients with ACS admitted consecutively between January 2004 and April 2007 to an urban academic cardiology emergency single-center in São Paulo, Brazil, were prospectively evaluated. The Ethics Committee of the hospital approved this study and written informed consent was obtained from all participants. Baseline clinical and admission laboratory characteristics, CAD risk factors, in-hospital mortality, and treatments were observed. Ejection fraction was obtained by echocardiography at the second day of hospital stay. A total of 1304 ACS patients were included, and a subgroup of 959 patients had complete data to be studied. Inclusion criteria were ACS hospital admission, according to the international consensus definition, age older than 18 years, and willing to provide written informed consent. Exclusion criteria were incomplete laboratory data. A sample size of at least 118 patients, with 59 subjects per treatment group, was determined to give $80 \%$ power to detect a $6 \%$ difference in the incidence of cardiovascular events with a $5 \%$ significance level.

\section{Definitions and data collection}

Clinical outcome was defined as death. Because $90 \%$ of patients at our emergency department undergo angiographic study in 48 hours, reinfarction or refractory angina are infrequent events at hospitalization. Blood samples were collected at admission to the emergency department and ejection fraction was obtained at second day of stay by echocardiography with the biplane Simpson's method. We selected the level of hyperglycaemia above $200 \mathrm{mg} / \mathrm{dL}$ to separate the highest stratum seen in the literature [13] for admission nonfasting blood glucose.

Patients were grouped by gender and ABG below ("moderate hyperglycaemia" = G-) or above ("higher hyperglycaemia" $=\mathrm{G}+) 200 \mathrm{mg} / \mathrm{dL}:$ menG- = men with glucose $\leq 200 \mathrm{mg} / \mathrm{dL}$; womenG- = women with glucose $\leq$ $200 \mathrm{mg} / \mathrm{dL}$; menG + = men with glucose $\geq 200 \mathrm{mg} / \mathrm{dL}$; and womenG $+=$ women with glucose $\geq 200 \mathrm{mg} / \mathrm{dL}$. We observed patient's distribution in acute coronary syndromes. UA was defined by hospital admission with thoracic pain (or any equivalent) considered to be of myocardial ischemic origin, and non ST-elevation on electrocardiographic or myocardial necrosis marker changes at first consultation. NSTEMI was defined by symptoms of myocardial ischemic origin, myocardial necrosis marker elevation, and no ST-elevation at first electrocardiogram in the emergency department. STEMI was considered when symptoms of myocardial ischemic origin and ST-elevation segment on electrocardiogram were present [14]. ABG was managed based on emergency physicians routine: subcutaneous intermittent insulin doses until blood glucose below $140 \mathrm{mg} / \mathrm{dL}$, and point-of-care glucose control after first blood glucose measurement.

Treatments were defined as medical therapy only, when patients were not candidate to angiography or the invasive study did not result in surgical or percutaneous intervention, and percutaneous (angioplasty) or emergency coronary surgery.

\section{Statistical analysis}

All data are described as rates and frequencies or means with standard deviations, as appropriate. Differences in the distribution of select characteristics between patient groups were examined using the chi-square test and Fisher's exact test for categorical variables. The analysis was performed using the Student $t$ test for normally distributed continuous variables and the Mann-Whitney and Kruskal-Wallis tests for nonparametric variables. General linear models analyzed the hierarchical differences of 3 groups, instead of ANOVA of the NPARWAY1 approach. We built an adjusted model in multivariate regression to analyze the independent variables associated with in-hospital death: age, family history of CAD, prior coronary artery disease, hypertension, smoking, diabetes, categorical admission blood glucose $<$ or $\geq 200 \mathrm{mg} / \mathrm{dL}$, gender, ejection fraction and serum creatinine. This analysis was done for all ACS patients and for AU, NSTEMI, and STEMI patients. In ACS analysis, we added type of ACS presentation to the logistic model. Sex and categorical blood glucose were analyzed as a specific subgroup in the logistic model. We performed analysis by the stepwise approach, and variables with $\mathrm{p}$ value $\leq 0.10$ were included in the model. Alternatively, we performed an interaction analysis of gender, categorical admission blood glucose $<$ or $\geq 200 \mathrm{mg} / \mathrm{dL}$ and type of ACS presentation. Two-sided $\mathrm{p}$ values $<0.05$ were considered statistically significant. All statistical analyses were performed using SAS software version 9.2 for Windows (SAS Institute Inc., Cary, NC, USA).

\section{Results}

Table 1 shows a comparison between types of ACS. Proportion of males increased from UA to NSTEMI $(53.9 \%$ vs $63.2 \%, \mathrm{p}=0.010)$, UA to STEMI $(53.9 \%$ vs $71.7 \%, \mathrm{p}<0.001)$, and NSTEMI to STEMI $(63.2 \%$ vs 
Table 1 Univariate clinical and demographic comparison between unstable angina, non-ST elevation and ST-elevation myocardial infarction patients

\begin{tabular}{|c|c|c|c|c|}
\hline & $U^{*} *$ & NSTEMI & STEMI & p-valuet \\
\hline Patients, n (\%) & $304(31.7)$ & $443(46.2)$ & $212(22.1)$ & \\
\hline Male, n (\%) & $164(53.9)$ & $280(63.2)$ & $152(71.7)$ & $a, b, c$ \\
\hline Age (years) ₹ & $60.7 \pm 0.4$ & $63.3 \pm 11.9$ & $59.2 \pm 12.8$ & $\mathrm{a}, \mathrm{c}$ \\
\hline Prior coronary artery disease, $\mathrm{n}(\%)$ & $198(65.1)$ & $239(53.9)$ & $70(33.0)$ & $a, b, c$ \\
\hline Hypertension, n (\%) & $258(84.9)$ & $365(82.4)$ & $146(68.9)$ & $b, c$ \\
\hline Current smoker, n (\%) & $51(16.8)$ & $89(20.1)$ & $72(34.0)$ & $b, c$ \\
\hline Dyslipidemia, n (\%) & $171(56.2)$ & $228(51.5)$ & $67(31.6)$ & $b, c$ \\
\hline Diabetes mellitus, n (\%) & $104(34.2)$ & $167(37.7)$ & $55(25.9)$ & c \\
\hline Family history of coronary artery disease, n (\%) & $44(14.5)$ & $84(18.9)$ & $35(16.5)$ & Non-significant \\
\hline Hemoglobin (g/dL) & $13.7 \pm 1.6$ & $13.6 \pm 1.9$ & $14.2 \pm 1.8$ & $b, c$ \\
\hline Serum creatinine $(\mathrm{mg} / \mathrm{dL})$ & $1.2 \pm 1.0$ & $1.3 \pm 1.3$ & $1.1 \pm 0.6$ & a \\
\hline Troponin I (ng/mL) & $1.3 \pm 6.5$ & $20.6 \pm 34.5$ & $56.6 \pm 57.9$ & $a, b, c$ \\
\hline Cholesterol (mg/dL) & $185.6 \pm 48.3$ & $180.5 \pm 49.0$ & $173.5 \pm 44.0$ & $\mathrm{~b}$ \\
\hline Admission glucose $(\mathrm{mg} / \mathrm{dL})$ & $126.9 \pm 60.4$ & $136.7 \pm 72.1$ & $136.4 \pm 66.7$ & Non-significant \\
\hline Ejection fraction (\%)† & $56.6 \pm 14.0$ & $51.2 \pm 15.5$ & $49.4 \pm 12.9$ & $a, b$ \\
\hline Angiography, n (\%) & $289(95.1)$ & $421(95.0)$ & $212(100.0)$ & $b, c$ \\
\hline Medical therapy only, n (\%) & $138(45.4)$ & $147(33.2)$ & $23(10.8)$ & $a, b, c$ \\
\hline Percutaneous coronary intervention, n (\%) & $145(47.7)$ & $263(59.4)$ & $177(83.5)$ & $a, b, c$ \\
\hline Surgery, n (\%) & $21(6.9)$ & $35(7.9)$ & $14(6.6)$ & Non-significant \\
\hline Length of stay (days) & $4.6 \pm 8.1$ & $7.3 \pm 12.3$ & $4.8 \pm 5.8$ & $\mathrm{a}, \mathrm{c}$ \\
\hline Mortality, n (\%) & $5(1.6)$ & $22(5.0)$ & $8(3.8)$ & a \\
\hline
\end{tabular}

*UA = unstable angina; NSTEMI = non-ST elevation myocardial infarction; STEMI = ST elevation myocardial infarction; $+\mathrm{p}$-value: $\mathrm{a}=\mathrm{UA}$ versus NSTEMI significant; $\mathrm{b}=$ UA versus STEMI significant; $c=$ NSTEMI versus STEMI significant; $\neq$ continuous variables are shown as mean (standard deviation), and categorical variables are shown as number (percentage).

$71.7 \%, \mathrm{p}=0.035)$. STEMI patients were younger than NSTEMI $(\mathrm{p}<0.001)$, had lower prior coronary artery disease than NSTEMI or UA $(p<0.001)$, hypertension ( $p$ $<0.001)$, dyslipidemia $(\mathrm{p}<0.001)$, diabetes $(\mathrm{p}=0.003)$, total cholesterol $(\mathrm{p}=0.009)$, ejection fraction $(\mathrm{p}<0.001$ in comparison with UA); more of them were current smokers $(\mathrm{p}<0.001)$ than UA or NSTEMI patients.

Of all patients (959), men totaled 596 and women 363 (UA 304, NSTEMI 443, and STEMI 212 patients). In STEMI patients, only 12 were treated with thrombolytic drugs and 200 with primary angioplasty. Table 2 presents clinical and demographic patient characteristics of all ACS, UA, NSTEMI and STEMI patients, grouped by $\mathrm{ABG}<$ or $\geq 200 \mathrm{mg} / \mathrm{dL}$ and gender. Univariate analysis showed that higher ABG patients were older $(\mathrm{p}<0.001)$, had more hypertension $(\mathrm{p}<0.001)$, diabetes $(\mathrm{p}<0.001)$, dyslipidemia $(p=0.002)$, higher mortality $(p<0.001)$ and length of stay $(p=0.003)$, less smoking $(p=0.008)$ and lower hemoglobin levels $(\mathrm{p}<0.001)$, needed more surgical interventions $(\mathrm{p}=0.046)$, and fewer percutaneous coronary interventions $(\mathrm{p}=0.038)$. Men had higher troponin $(p=0.003)$ and hemoglobin levels $(p<0.001)$, but lower total cholesterol $(\mathrm{p}<0.001)$ and ejection fraction $(\mathrm{p}<0.001)$ than women had. In unstable angina, higher
ABG patients had more prior CAD ( $\mathrm{p}=0.013)$, hypertension $(p=0.019)$, and diabetes $(p<0.001)$. In NSTEMI, higher ABG patients were older $(p=0.006)$, had more hypertension $(p=0.026)$, diabetes $(p<0.001)$, higher length of stay $(p=0.002)$, mortality $(p<0.001)$, lower ejection fraction $(\mathrm{p}<0.001)$, angiography $(\mathrm{p}=0.028)$, and percutaneous coronary intervention $(p=0.006)$. In STEMI, higher ABG patients had more hypertension $(\mathrm{p}=0.009)$, diabetes $(p<0.001)$, mortality $(p=0.012)$, and less smoking $(\mathrm{p}=0.036)$.

In multivariate logistic analysis, the adjusted model showed as independent factors related to ACS in-hospital death, age $(\mathrm{OR}=1.057$, IC 95\% $1.022-1.093$; $\mathrm{p}=0.001)$, serum creatinine level $(\mathrm{OR}=1.337$, IC 95\% 1.335 - 1.574; $\mathrm{p}<0.001)$, ejection fraction $(\mathrm{OR}=0.943$, IC $95 \% 0.917-$ $0.970 ; \mathrm{p}<0.001$ ), as showed in Figure 1. When interaction between gender and categorical admission blood glucose was adjusted by logistic model, womenG- had lower mortality than menG + (OR 0.259, IC 95\% 0.074 - 0.905; $\mathrm{p}=0.034)$, but no difference with womenG $+(\mathrm{OR}$ 0.312 , IC 95\% $0.086-1.130 ; \mathrm{p}=0.076)$. Group menGhad lower mortality than menG + (OR 0.160, IC 95\% $0.057-0.452 ; \mathrm{p}<0.001)$ or womenG + (OR 0.193, IC $95 \% 0.063-0.588 ; \mathrm{p}=0.004)$. There was no difference 
Table 2 Clinical and demographic characteristics of acute coronary syndrome, unstable angina, non-ST elevation, and ST-elevation myocardial infarction patients, grouped by gender and glycaemia glucose $<$ or $\geq 200 \mathrm{mg} / \mathrm{dL}$

\begin{tabular}{|c|c|c|c|c|c|}
\hline $\mathrm{ACS}^{*}$ & $\begin{array}{l}\text { Men glucose } \\
<200\end{array}$ & $\begin{array}{l}\text { Women glucose } \\
<200\end{array}$ & $\begin{array}{l}\text { Men glucose } \\
\geq 200\end{array}$ & $\begin{array}{l}\text { Women glucose } \\
\geq 200\end{array}$ & $p+t$ \\
\hline Patients & $454(47.3)$ & $242(25.2)$ & $142(14.8)$ & $121(12.6)$ & \\
\hline Age (years) $†$ & $60.1 \pm 11.4$ & $61.9 \pm 11.7$ & $63.1 \pm 11.3$ & $64.6 \pm 12.9$ & $<0.001$ \\
\hline Prior coronary artery disease, $\mathrm{n}(\%)$ & $244(53.7)$ & $113(46.7)$ & $81(57.0)$ & $69(57.0)$ & 0,129 \\
\hline Hypertension, n (\%) & $338(74.4)$ & $197(81.4)$ & $122(85.9)$ & $112(92.6)$ & $<0.001$ \\
\hline Current smoker, n (\%) & $119(26.2)$ & $53(21.9)$ & $23(16.2)$ & $17(14.0)$ & 0,008 \\
\hline Dyslipidemia, n (\%) & $192(42.3)$ & $133(55.0)$ & $73(51.4)$ & $68(56.2)$ & 0,002 \\
\hline Diabetes mellitus, n (\%) & $80(17.6)$ & $50(20.7)$ & $103(72.5)$ & $93(76.9)$ & $<0.001$ \\
\hline Family history of coronary artery disease, n (\%) & $77(17.0)$ & $40(16.5)$ & $23(16.2)$ & $23(19.0)$ & 0,929 \\
\hline Hemoglobin (g/dL) & $14.3 \pm 1.8$ & $13.2 \pm 1.5$ & $13.8 \pm 1.9$ & $12.9 \pm 1.8$ & $<0.001$ \\
\hline Serum creatinine $(\mathrm{mg} / \mathrm{dL})$ & $1.2 \pm 0.8$ & $1.1 \pm 1.3$ & $1.3 \pm 1.0$ & $1.3 \pm 1.4$ & 0,331 \\
\hline Troponin I (ng/mL) & $25.4 \pm 44.4$ & $14.8 \pm 32.9$ & $25.0 \pm 44.7$ & $16.6 \pm 31.8$ & 0,003 \\
\hline Cholesterol (mg/dL) & $175.6 \pm 44.6$ & $194.5 \pm 54.3$ & $166.5 \pm 43.9$ & $188.6 \pm 43.1$ & $<0.001$ \\
\hline Admission glucose (mg/dL) & $101.6 \pm 15.8$ & $100.0 \pm 14.9$ & $211.2 \pm 67.7$ & $229.0 \pm 84.1$ & $<0.001$ \\
\hline Ejection fraction (\%) & $50.9 \pm 14.2$ & $58.2 \pm 13.3$ & $46.8 \pm 14.9$ & $51.9 \pm 15.6$ & $<0.001$ \\
\hline Angiography, n (\%) & $445(98.0)$ & $229(94.6)$ & $133(93.7)$ & $115(95.0)$ & 0,036 \\
\hline Medical therapy only, n (\%) & $134(29.5)$ & $88(36.4)$ & $42(29.6)$ & $44(36.4)$ & 0,184 \\
\hline Percutaneous coronary intervention, n (\%) & $298(65.6)$ & $137(56.6)$ & $84(59.2)$ & $66(54.6)$ & 0,038 \\
\hline Surgery, n (\%) & $23(5.1)$ & $19(7.8)$ & $16(11.3)$ & $12(9.9)$ & 0,046 \\
\hline Length of stay (days) & $5.0 \pm 6.6$ & $5.7 \pm 10.5$ & $8.2 \pm 16.1$ & $7.2 \pm 9.6$ & 0,003 \\
\hline Mortality, n (\%) & $6(1.3)$ & $4(1.6)$ & $15(10.6)$ & $10(8.3)$ & $<0.001$ \\
\hline \multicolumn{6}{|l|}{ UA } \\
\hline Patients, n (\%) & $132(43.4)$ & $100(32.9)$ & $32(10.5)$ & $40(13.2)$ & \\
\hline Age (years)* & $59.9 \pm 10.9$ & $60.7 \pm 9.6$ & $61.6 \pm 10.7$ & $62.5 \pm 10.8$ & 0.537 \\
\hline Prior coronary artery disease, $\mathrm{n}(\%)$ & $91(68.9)$ & $55(55.0)$ & $27(84.4)$ & $25(62.5)$ & 0.013 \\
\hline Hypertension, n (\%) & $103(78.0)$ & $88(88.0)$ & $31(96.9)$ & $36(90.0)$ & 0.019 \\
\hline Current smoker, n (\%) & $25(18.9)$ & $16(16.0)$ & $6(18.7)$ & $4(10.0)$ & 0.595 \\
\hline Dyslipidemia, n (\%) & $68(51.5)$ & $65(65.0)$ & $17(53.1)$ & $21(52.5)$ & 0.197 \\
\hline Diabetes mellitus, n (\%) & $27(20.5)$ & $23(23.0)$ & $24(75.0)$ & $30(75.0)$ & $<0.001$ \\
\hline Family history of coronary artery disease, n (\%) & $22(16.7)$ & $14(14.0)$ & $4(12.5)$ & $4(10.0)$ & 0.734 \\
\hline Hemoglobin (g/dL) & $14.1 \pm 1.7$ & $13.3 \pm 1.4$ & $13.9 \pm 1.9$ & $13.5 \pm 1.5$ & 0.002 \\
\hline Serum creatinine $(\mathrm{mg} / \mathrm{dL})$ & $1.3 \pm 1.2$ & $0.9 \pm 0.3$ & $1.2 \pm 0.5$ & $1.3 \pm 1.5$ & 0.032 \\
\hline Troponin I (ng/mL) & $1.0 \pm 3.7$ & $0.5 \pm 1.6$ & $1.2 \pm 3.5$ & $3.7 \pm 16.0$ & 0.067 \\
\hline Cholesterol (mg/dL) & $176.3 \pm 44.1$ & $193.9 \pm 46.7$ & $178.6 \pm 63.5$ & $200.5 \pm 48.9$ & 0.017 \\
\hline Admission glucose (mg/dL) & $99.6(14.6)$ & $99.3(15.4)$ & $222.5(66.3)$ & $209.5(67.3)$ & $<0.001$ \\
\hline Ejection fraction (\%)† & $56.1 \pm 13.7$ & $59.5 \pm 12.8$ & $50.7 \pm 15.5$ & $55.5 \pm 15.2$ & 0.069 \\
\hline Angiography, n (\%) & $127(96.2)$ & $93(93.0)$ & $30(93.8)$ & $39(97.5)$ & 0.593 \\
\hline Medical therapy only, n (\%) & $57(43.2)$ & $53(53.0)$ & $11(34.4)$ & $17(42.5)$ & 0.231 \\
\hline Percutaneous coronary intervention, n (\%) & $67(50.8)$ & $40(40.0)$ & $17(53.1)$ & $21(52.5)$ & 0.306 \\
\hline Surgery, n (\%) & $8(6.1)$ & $7(7.0)$ & $4(12.5)$ & $2(5.0)$ & 0.587 \\
\hline Length of stay (days) & $4.9 \pm 9.1$ & $3.5 \pm 3.2$ & $7.3 \pm 14.6$ & $4.2 \pm 4.9$ & 0.115 \\
\hline Mortality, n (\%) & $1(0.8)$ & 0 & $4(12.5)$ & 0 & $<0.001$ \\
\hline \multicolumn{6}{|l|}{ NSTEMI } \\
\hline Patients, n (\%) & $208(46.9)$ & $103(23.3)$ & $72(16.3)$ & $60(13.5)$ & \\
\hline Age (years)* & $61.2 \pm 11.4$ & $64.4 \pm 12.9$ & $65.7 \pm 10.3$ & $65.7 \pm 12.5$ & 0.006 \\
\hline Prior coronary artery disease, $\mathrm{n}(\%)$ & $115(55.3)$ & $46(44.7)$ & $42(58.3)$ & $36(60.0)$ & 0.159 \\
\hline
\end{tabular}


Table 2 Clinical and demographic characteristics of acute coronary syndrome, unstable angina, non-ST elevation, and ST-elevation myocardial infarction patients, grouped by gender and glycaemia glucose $<$ or $\geq \mathbf{2 0 0} \mathbf{~ m g / d L}$ (Continued)

\begin{tabular}{|c|c|c|c|c|c|}
\hline Hypertension, $\mathrm{n}(\%)$ & $161(77.4)$ & $85(82.5)$ & $64(88.9)$ & $55(91.7)$ & 0.026 \\
\hline Current smoker, n (\%) & $50(24.0)$ & $21(20.4)$ & $7(9.7)$ & $11(18.3)$ & 0.073 \\
\hline Dyslipidemia, n (\%) & $97(46.6)$ & $53(51.5)$ & $41(56.9)$ & $37(61.70$ & 0.151 \\
\hline Diabetes mellitus, n (\%) & $41(19.7)$ & $23(22.3)$ & $55(76.4)$ & $48(80.0)$ & $<0.001$ \\
\hline Family history of coronary artery disease, n (\%) & $37(17.8)$ & $19(18.4)$ & $14(19.4)$ & $14(23.3)$ & 0.811 \\
\hline Hemoglobin $(\mathrm{g} / \mathrm{dL})$ & $14.3 \pm 1.8$ & $13.0 \pm 1.6$ & $13.2 \pm 2.0$ & $12.7 \pm 1.9$ & $<0.001$ \\
\hline Serum creatinine $(\mathrm{mg} / \mathrm{dL})$ & $1.2 \pm 0.7$ & $1.3 \pm 1.9$ & $1.5 \pm 1.4$ & $1.4 \pm 1.5$ & 0.586 \\
\hline Troponin I (ng/mL) & $22.6 \pm 36.9$ & $18.4 \pm 34.4$ & $19.5 \pm 31.2$ & $18.9 \pm 29.7$ & 0.734 \\
\hline Cholesterol (mg/dL) & $178.4 \pm 47.2$ & $194.5 \pm 61.2$ & $167.1 \pm 38.0$ & $182.6 \pm 39.8$ & 0.011 \\
\hline Admission glucose (mg/dL) & $100.8 \pm 16.5$ & $101.9 \pm 14.8$ & $202.7 \pm 62.6$ & $241.3 \pm 98.7$ & $<0.001$ \\
\hline Ejection fraction (\%)† & $49.8 \pm 14.9$ & $59.3 \pm 13.5$ & $44.7 \pm 14.9$ & $49.0 \pm 16.0$ & $<0.001$ \\
\hline Angiography, n (\%) & $204(98.1)$ & $97(94.2)$ & $65(90.3)$ & $55(91.7)$ & 0.028 \\
\hline Medical therapy only, n (\%) & $63(30.3)$ & $31(30.1)$ & $27(37.5)$ & $26(43.3)$ & 0.202 \\
\hline Percutaneous coronary intervention, n (\%) & $135(64.9)$ & $66(64.1)$ & $36(50.0)$ & $26(43.3)$ & 0.006 \\
\hline Surgery, n (\%) & $10(4.8)$ & $8(7.8)$ & $9(12.5)$ & $8(13.3)$ & 0.064 \\
\hline Length of stay (days) & $5.1 \pm 5.2$ & $7.7 \pm 14.5$ & $10.7 \pm 20.1$ & $9.9 \pm 12.4$ & 0.002 \\
\hline Mortality, n (\%) & $4(1.9)$ & $3(2.9)$ & $8(11.1)$ & $7(11.7)$ & $<0.001$ \\
\hline \multicolumn{6}{|l|}{ STEMI } \\
\hline Patients, n (\%) & $114(53.8)$ & $39(18.4)$ & $38(17.9)$ & $21(9.9)$ & \\
\hline Age (years)* & $58.1 \pm 11.9$ & $58.7 \pm 12.3$ & $59.3 \pm 12.5$ & $65.7 \pm 17.1$ & 0.096 \\
\hline Prior coronary artery disease, $\mathrm{n}(\%)$ & $38(33.3)$ & $12(30.8)$ & $12(31.6)$ & $8(38.1)$ & 0.945 \\
\hline Hypertension, n (\%) & $74(64.9)$ & $24(61.5)$ & $27(71.1)$ & $21(100)$ & 0.009 \\
\hline Current smoker, n (\%) & $44(38.6)$ & $16(41.0)$ & $10(26.3)$ & $2(9.5)$ & 0.036 \\
\hline Dyslipidemia, n (\%) & $27(23.7)$ & $15(38.5)$ & $15(39.5)$ & $10(47.6)$ & 0.052 \\
\hline Diabetes mellitus, n (\%) & $12(10.5)$ & $4(10.3)$ & $24(63.2)$ & $15(71.4)$ & $<0.001$ \\
\hline Family history of coronary artery disease, n (\%) & $18(15.8)$ & $7(17.9)$ & $5(13.2)$ & $5(23.8)$ & 0.748 \\
\hline Hemoglobin (g/dL) & $14.5 \pm 1.8$ & $13.2 \pm 1.6$ & $14.8 \pm 1.4$ & $12.7 \pm 2.1$ & $<0.001$ \\
\hline Serum creatinine $(\mathrm{mg} / \mathrm{dL})$ & $1.2 \pm 0.4$ & $1.1 \pm 1.0$ & $1.1 \pm 0.4$ & $1.1 \pm 0.5$ & 0.758 \\
\hline Troponin I (ng/mL) & $58.8 \pm 59.4$ & $41.0 \pm 48.7$ & $56.1 \pm 65.3$ & $34.7 \pm 47.8$ & 0.173 \\
\hline Cholesterol (mg/dL) & $169.3 \pm 39.4$ & $195.9 \pm 56.6$ & $156.5 \pm 34.7$ & $180.7 \pm 36.1$ & 0.001 \\
\hline Admission glucose $(\mathrm{mg} / \mathrm{dL})$ & $105.4 \pm 15.4$ & $96.7 \pm 13.6$ & $217.9 \pm 77.2$ & $230.9 \pm 60.3$ & $<0.001$ \\
\hline Ejection fraction (\%)† & $47.9 \pm 12.0$ & $51.5 \pm 12.1$ & $47.9 \pm 14.6$ & $55.4 \pm 13.8$ & 0.098 \\
\hline Angiography, n (\%) & $114(100.0)$ & $39(100.0)$ & $38(100.0)$ & $21(100.0)$ & 1.000 \\
\hline Medical therapy only, n (\%) & $14(12.3)$ & $4(10.3)$ & $4(10.5)$ & $1(4.8)$ & 0.786 \\
\hline Percutaneous coronary intervention, n (\%) & $96(84.2)$ & $31(79.5)$ & $31(81.6)$ & $19(90.5)$ & 0.719 \\
\hline Surgery, n (\%) & $5(4.4)$ & $4(10.3)$ & $3(7.9)$ & $2(9.5)$ & 0.543 \\
\hline Length of stay (days) & $4.6 \pm 5.3$ & $5.9 \pm 9.2$ & $4.2 \pm 3.4$ & $4.9 \pm 2.8$ & 0.546 \\
\hline Mortality, n (\%) & $1(0.9)$ & $1(2.6)$ & $3(7.9)$ & $3(14.3)$ & 0.012 \\
\hline
\end{tabular}

${ }^{*} \mathrm{ACS}$ = acute coronary syndrome; UA = unstable angina; NSTEMI = non-ST elevation myocardial infarction; STEMI = ST elevation myocardial infarction; +continuous variables are shown as mean (standard deviation), and categorical variables are shown as number (percentage); †† $p$-values refers to four-groups comparisons.

between gender in admission blood glucose $<200 \mathrm{mg} / \mathrm{dL}$ (OR 0.618, IC 95\% $0.0156-2.449 ; \mathrm{p}=0.494$ ) or $\geq$ $200 \mathrm{mg} / \mathrm{dL}$ (OR 1.202, IC 95\% 0.470 - 3.075; p = 0.701).

When patients were separated by ACS type, in UA group we observed that age (OR 1.168, IC 95\% 1.024 - 1.332; $\mathrm{p}=0.021)$ and admission blood glucose (OR 1.019, IC 95\% $1.007-1.031 ; \mathrm{p}=0.002$ ) were independent factors related to in-hospital death, whereas in NSTEMI we had age (OR 1.090, IC 95\% $1.038-1.144 ; \mathrm{p}<0.001)$, ejection fraction (OR 0.942, IC 95\% $0.908-0.977 ; \mathrm{p}=0.001$ ), creatinine (OR 1.357, IC 95\% $1.124-1.639 ; \mathrm{p}=0.001)$, and admission blood glucose (OR 1.005, IC 95\% $1.000-1.009 ; \mathrm{p}=0.037$ ). Finally, in the STEMI group we found creatinine (OR 13.446, IC 95\% $2.840-63.656 ; \mathrm{p}=0.001$ ), and female 


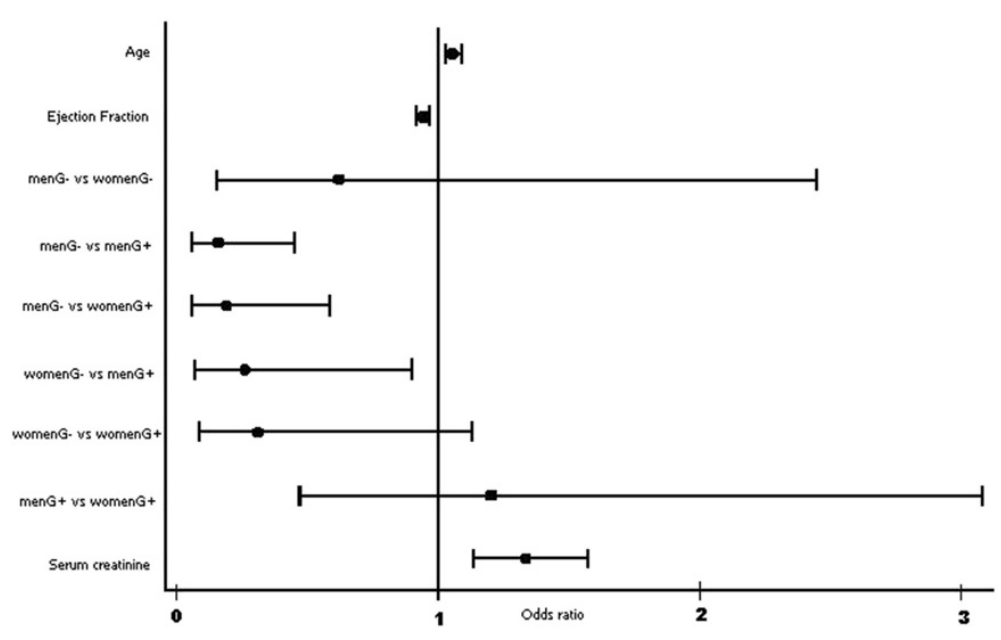

Figure 1 Odds ratio of in-hospital adjusted mortality of groups gender and hyperglycaemia; menG- = men with moderate hyperglycaemia $(<200 \mathrm{mg} / \mathrm{dL})$; womenG- = Women with moderate hyperglycaemia $(<200 \mathrm{mg} / \mathrm{dL})$; $\mathrm{menG}+=$ Men with higher hyperglycaemia ( $\geq 200 \mathrm{mg} / \mathrm{dL}$ ); womenG $+=$ Women with higher hyperglycaemia ( $\geq 200 \mathrm{mg} / \mathrm{dL}$ ).

gender (OR 0.147, IC 95\% 0.024 - 0.886; $\mathrm{p}=0.036$ for men versus women) as independent risks for death, as showed in Table 3.

In interaction analysis, multivariate logistic analysis model showed age (OR 1.060, IC 95\% 1.025 - 1.096; $\mathrm{p}<0.001$ ), ejection fraction (OR 0.944, IC 95\% 0.918 - 0.971; p $<0.001$ ), serum creatinine (OR 1.356, IC 95\% 1.151 - 1.598; $\mathrm{p}<0.001)$ and interaction $\mathrm{ABG}<200 \mathrm{mg} / \mathrm{dL}$ in male gender (OR 0.171, IC 95\% $0.061-0.478 ; \mathrm{p}=0.017$ in comparison to $A B G \geq 200 \mathrm{mg} / \mathrm{dL}$ ). In female gender, categorical blood glucose $<$ or $\geq 200 \mathrm{mg} / \mathrm{dL}$ had no difference (OR 0.321, IC 95\% 0.090 - 1.151; $\mathrm{p}=0.078$ ).

Table 3 Odds ratio of in-hospital adjusted mortality for unstable angina, non-ST elevation myocardial infarction, and ST elevation myocardial infarction

\begin{tabular}{lllll}
\hline UA* & Odds Ratio & $\begin{array}{l}\text { Inferior limit } \\
\mathbf{9 5 \%} \text { IC }\end{array}$ & $\begin{array}{l}\text { Superior limit } \\
\mathbf{9 5 \%} \text { IC }\end{array}$ & $\mathbf{p}$ \\
\hline Age & 1.168 & 1.024 & 1.332 & 0.021 \\
\hline $\begin{array}{l}\text { Admission blood } \\
\text { glucose }\end{array}$ & 1.019 & 1.007 & 1.031 & 0.002 \\
\hline
\end{tabular}

\begin{tabular}{lllll}
\hline NSTEMI & & & \\
\hline Age & 1.090 & 1.038 & 1.144 & $<0.001$ \\
\hline Ejection fraction & 0.942 & 0.908 & 0.977 & 0.001 \\
\hline Creatinine & 1.357 & 1.124 & 1.639 & 0.001 \\
\hline $\begin{array}{l}\text { Admission blood } \\
\text { glucose }\end{array}$ & 1.005 & 1.000 & 1.009 & 0.037 \\
\hline STEMI & & & & \\
\hline Ejection fraction & 0.939 & 0.878 & 1.005 & 0.069 \\
\hline Creatinine & 13.446 & 2.840 & 63.656 & 0.001 \\
\hline Men vs Women & 0.147 & 0.024 & 0.886 & 0.036
\end{tabular}

* UA = unstable angina; NSTEMI = non-ST elevation myocardial infarction; STEMI = ST elevation myocardial infarction; IC = interval of confidence.
Figure 2 shows interaction between gender and categorical blood glucose group.

\section{Discussion}

The results of this study show that ABG influences men's in-hospital mortality from ACS, but this effect was not observed in women when patients of the same gender and different degrees of ABG were compared. According to ACS type of presentation, ABG was an independent factor related to in-hospital mortality in UA and NSTEMI, but not with STEMI in this study population. In fact, differences between diagnostic categories can exist, like that found in the Falciglia et al study [8], a significant association between hyperglycaemia and adjusted mortality was seen in unstable angina, acute myocardial infarction, congestive heart failure, arrhythmia, ischemic and hemorrhagic stroke, gastrointestinal bleeding, acute renal failure, pneumonia, pulmonary embolism and sepsis, but not in patients with chronic obstructive pulmonary disease, hepatic failure, and gastrointestinal neoplasm, or those admitted to the intensive care unit after surgery for coronary artery bypass graft, peripheral vascular disease, and hip fracture. But in our study, ABG was not an independent factor in STEMI, and this is different from other studies $[4,7]$. Lower age of STEMI patients in our study could explain both the similarity in mortality between STEMI and NSTEMI groups and the absence of the influence of glucose on STEMI mortality.

Several hypotheses have been raised to explain the role of hyperglycaemia in the worse prognosis in ACS. First, ABG could reflect uncontrolled or undiagnosed diabetes and a more advanced atherosclerosis. To justify this point of view, there is an association of $\mathrm{ABG}$ with elevation of 


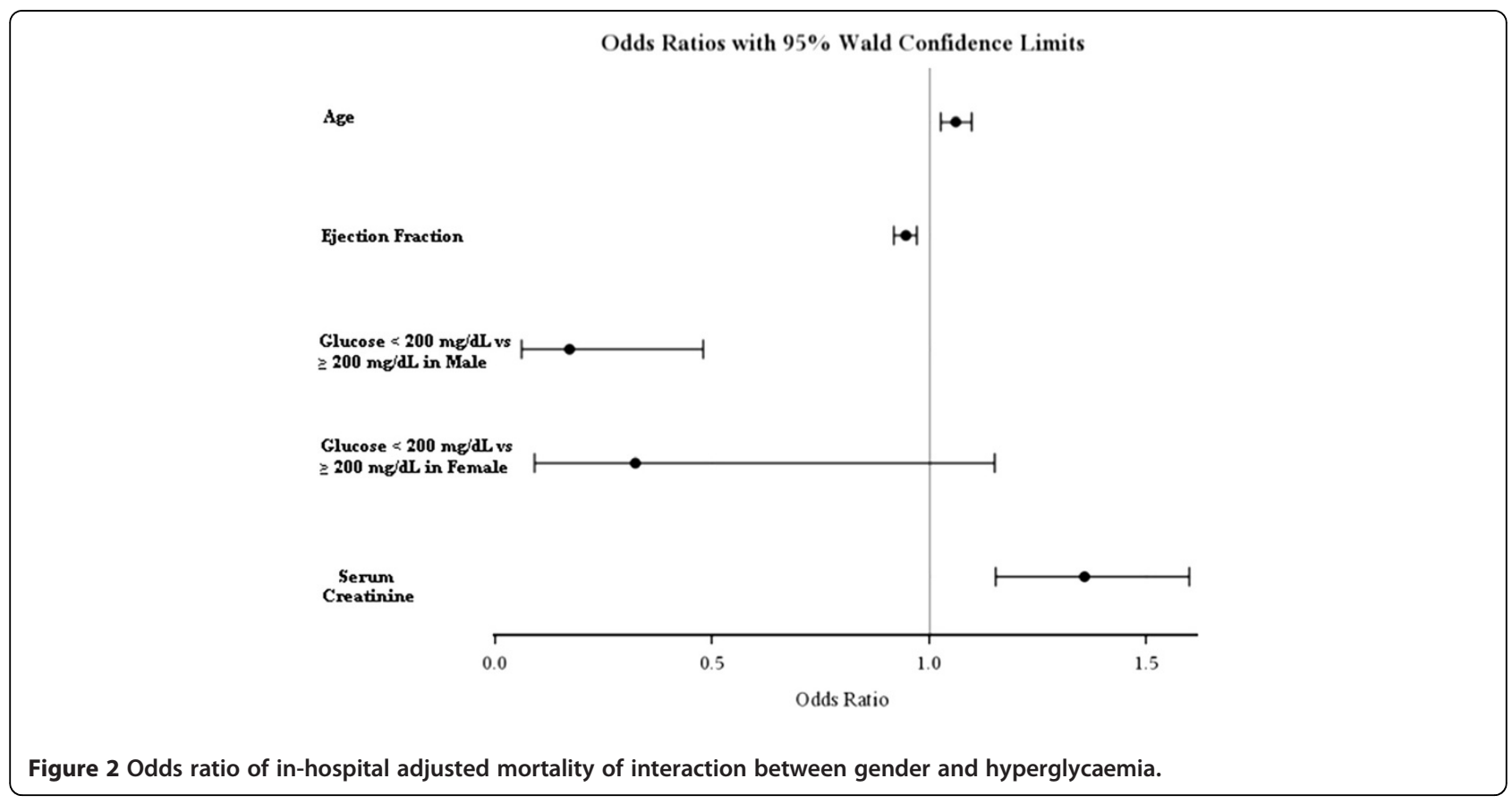

hemoglobin A1C in these patients [15,16]. Second, ABG is a common acute adrenergic signal of stress and is present in myocardial infarction [17], as well as other severe acute illnesses [8], whereas increased catecholamine levels result in decreased insulin secretion and increased insulin resistance [18]. Third, several studies show that hyperglycaemia could by itself be harmful to ischemic myocardium. For example, acute increases in plasma glucose levels have significant hemodynamic effects, even in normal subjects. In one study, maintenance of plasma glucose levels at $15.0 \mathrm{mmol} / \mathrm{L}(270 \mathrm{mg} / \mathrm{dL})$ for 2 hours in healthy subjects significantly increased mean heart rate $(+9$ beats per minute; $\mathrm{p}<0.010)$, systolic (+20 mmHg; $\mathrm{p}<0.010)$ and diastolic blood pressure $(+14 \mathrm{mmHg}, \mathrm{p}<0.001)$ and plasma catecholamine levels [19]. In another study of flow-mediated endothelium-dependent vasodilatation of the brachial artery, significant decreases were found at one and two hours in those with impaired glucose tolerance or diabetes, but not in control subjects [20]. Other mechanisms of cell injury by hyperglycaemia include higher inflammatory response, higher circulating intercellular adhesion molecule (ICAM)- 1 and increased production of superoxide radicals and other reactive oxygen species by oxidative stress [21]. Magnetic resonance studies [22] show that hyperglycemic or diabetic patients have greater myocardial infarct size [23], leading to impaired blood nutrition to the ischemic myocardial wall. Jensen et al [24] showed that hyperglycaemia at admission in STEMI patients who are successfully treated by percutaneous angioplasty is independently associated with the presence and extent of microvascular obstruction on contrast-enhanced magnetic resonance. Thus, microvascular obstruction as assessed by magnetic resonance may be a mechanism that relates ABG in acute STEMI to a worse outcome.

The results of admission blood glucose are concordant with others hyperglycaemia and insulin resistance evaluations in ACS patients. Sinnaeve et al [25] showed that both elevated fasting glucose and admission blood glucose worse in-hospital and 6-month mortality in ACS patients. Greater glucose level (fasting or admission), higher risk of in-hospital death, congestive heart failure, and cardiogenic shock. Su et al [26] studied glycaemic variability determined by a continuous 72 -hours glucose monitoring system and the presence and severity of coronary artery disease in patients with type 2 diabetes mellitus. They found a correlation between mean amplitude of glycaemic excursions (difference between peaks and nadirs of glycaemia) and Gensini score $(r=0.277 ; p<0.001)$, which assesses the severity of coronary artery disease: it grades narrowing of the lumen of the coronary artery and scores it as 1 for $1-25 \%$ narrowing, 2 for $26-50 \%$ narrowing, 4 for $51-75 \%, 8$ for $76-90 \%, 16$ for $91-99 \%$ and 32 for a completely occluded artery. Feinberg et al [27] compared 30-day and 1-year mortality in non-diabetic ACS patients, groups of patients with (359 patients) and without (701 patients) metabolic syndrome criteria. Hyperglycaemia increases 30-day mortality risk only in metabolic syndrome patients, but 1-year mortality was increased by hyperglycaemia in both groups (metabolic or non-metabolic syndrome patients). Or 
in impaired glucose tolerance ACS patients, longterm mortality is higher in patients with $2 \mathrm{~h}$ postload hyperglycemia of $\geq 160 \mathrm{mg} / \mathrm{dL}$, and equal to previously known diabetes [28]. Even in non diabetic patients with acute decompensated heart failure admissions without myocardial infarction, hyperglycaemia increases short and long-term mortality [29], maybe reflecting the same higher activation of the sympathetic system. In this setting, it is interesting the correlation of ABG with B-type brain natriuretic factor [30], a well-known heart failure prognostic factor.

Based on these results, how could we explain the absence of effect, or at least absence of linear effect, of hyperglycaemia in women's SCA mortality? One possibility could be women's comparative lower inflammatory response. Several studies show the influence of gender on inflammation. Schroder et al. [31] showed that women had lower TNF and higher IL-10 levels in sepsis. Oberholzer et al. [32] found that male trauma patients had higher IL-6 levels than women. Deshpande and colleagues [33] showed that estradiol attenuated the LPS-induced production of IL-1, IL-6, and TNF by macrophages and also decreased NFkB-binding activity. But some confounder factors can interfere in the results of studies of ACS patients. Women ACS patients are older than men in most of trials and databases, and older people also have higher hyperglycaemia, insulin resistance, erythrocyte sedimentation rate $[34,35]$ like women. In other words, statistical adjustment for these confounder factors could not be enough.

Female STEMI adjusted in-hospital mortality persisted high, like other's observations $[9,10,36]$. Vaccarino and co-workers [10] studied NRMI data and showed an increased risk in women for death even after coronary disease risk factor adjustment, except in patients over 75 years old, perhaps related to the comparative delay in hospital arrival, delay in diagnosis, and lower probability of receiving thrombolytic therapy. But 10 years later [12], the same group found that at ages 50 to 70 the higher adjusted mortality in women persisted, but not at other ages. So, they hypothesized that this was the result of greater awareness of heart disease in younger women in recent years or improved diagnosis or treatment of acute coronary syndromes [11]. This improvement in women's cardiovascular mortality was observed in a study of a Brazilian population [37].

\section{Study limitations}

This study assesses urban Brazilian ACS patients at a specialized cardiology center. Of STEMI patients, only 12 were treated with thrombolytics drugs, a situation not present in nonspecialized centers. We did not evaluate long-term outcomes, or assess risk scores like GRACE or TIMI.

\section{Conclusions}

We conclude that age and ABG were independent risk factors for death in UA; age, lower ejection fraction, higher creatinine and $A B G$ were independent risk factors for death in NSTEMI; and lower ejection fraction, higher creatinine and female gender were independent risk factors for death in STEMI. In global ACS patients, ABG influenced prognosis negatively in men, but this relation was not found in women, when hyperglycaemia was compared in the same gender.

\section{Competing interests}

Conflicts of Interest and Source of Funding: Authors declare no conflicts of interest. This study was partially supported by FAPESP and CNPq.

\section{Authors' contribution}

JYT cared out paper conception, writing, data collection and statistical analysis. RBR, LCR, SDA participated in data collection. JAFR participated in data analysis and review process. APM carried out paper conception, data collection and analysis. All authors read and approved the final manuscript.

Received: 8 February 2012 Accepted: 3 May 2012

Published: 3 May 2012

\section{References}

1. Van de Werf F, Bax J, Betriu A, Blomstrom-Lundqvist C, Crea F, Falk V, Filippatos G, Fox K, Huber K, Kastrati A, Rosengren A, Steg PG, Tubaro M, Verheugt F, Weidinger F, Weis M: ESC Committee for Practice Guidelines (CPG). Management of acute myocardial infarction in patients presenting with persistent ST-segment elevation: the Task Force on the Management of ST-Segment Elevation Acute Myocardial Infarction of the European Society of Cardiology. Eur Heart J 2008, 29:2909-2945.

2. Ramos RB, Strunz CM, Avakian SD, Ramires JA, Mansur AP: B-type natriuretic peptide as a predictor of anterior wall location in patients with non-STelevation myocardial infarction. Clinics (São Paulo) 2011, 66:437-441.

3. Modan B, Schor S, Shani M: Acute myocardial infarction. Prognostic value of white blood cell count and blood glucose level. JAMA 1975, 233:266-267.

4. Straumann E, Kurz DJ, Muntwyler J, Stettler I, Furrer M, Naegeli B, Frielingsdorf J, Schuiki E, Mury R, Bertel O, Spinas GA: Admission glucose concentrations independently predict early and late mortality in patients with acute myocardial infarction treated by primary or rescue percutaneous coronary intervention. Am Heart J 2005, 150:1000-1006.

5. Beck JA, Meisinger C, Heier M, Kuch B, Hörmann A, Greschik C, Koenig W: Effect of blood glucose concentrations on admission in non-diabetic versus diabetic patients with first acute myocardial infarction on shortand long-term mortality (from the MONICA/KORA Augsburg Myocardial Infarction Registry). Am J Cardiol 2009, 104:1607-1612.

6. Cid-Alvarez B, Gude F, Cadarso-Suarez C, Gonzalez-Babarro E, RodriguezAlvarez MX, Garcia-Acuna JM, Gonzalez-Juanatey JR: Admission and fasting plasma glucose for estimating risk of death of diabetic and nondiabetic patients with acute coronary syndrome: nonlinearity of hazard ratios and time-dependent comparison. Am Heart J 2009, 158:989-997.

7. Hoebers LP, Damman P, Claessen BE, Vis MM, Baan J Jr, van Straalen JP, Fischer J, Koch KT, Tijssen JG, de Winter RJ, Piek JJ, Henriques JP. Predictive value of plasma glucose level on admission for short and long term mortality in patients with ST-elevation myocardial infarction treated with primary percutaneous coronary intervention. Am J Cardiol 2011 Sep 23. [Epub ahead of print].

8. Falciglia M, Freyberg RW, Almenoff PL, D'Alessio DA, Render ML: Hyperglycemia-related mortality in critically ill patients varies with admission diagnosis. Crit Care Med 2009, 37:3001-3009.

9. Ineid $\mathrm{H}$, Fonarow GC, Cannon CP, Hernandez AF, Palacios IF, Maree AO, Wells Q, Bozkurt B, Labresh KA, Liang L, Hong Y, Newby LK, Fletcher G, Peterson E, Wexler L: Get With the Guidelines Steering Committee and Investigators. Sex differences in medical care and early death after acute myocardial infarction. Circulation 2008, 118:2803-2810.

10. Vaccarino V, Parsons L, Every NR, Barron HV, Krumholz HM: Sex-based differences in early mortality after myocardial infarction. National 
Registry of Myocardial Infarction 2 Participants. N Engl J Med 1999, 341:217-225.

11. Vaccarino V, Parsons L, Peterson ED, Rogers WJ, Kiefe Cl, Canto J: Sex differences in mortality after acute myocardial infarction: changes from 1994 to 2006. Arch Intern Med 2009, 169:1767-1774.

12. Champney KP, Frederick PD, Bueno H, Parashar S, Foody J, Merz CN, Canto $J G$, Lichtman JH, Vaccarino V: NRMI Investigators. The joint contribution of sex, age and type of myocardial infarction on hospital mortality following acute myocardial infarction. Heart 2009, 95:895-899.

13. Cubbon RM, Rajwani A, Abbas A, Gale CP, Grant PJ, Wheatcroft SB, Barth $\mathrm{JH}$, Kearney MT, Hall AS: Hyperglycaemia, in relation to sex, and mortality after acute coronary syndrome. Eur J Cardiovasc Prev Rehabil 2007, 14:666-671

14. Alpert JS, Thygesen K, Antman E, Bassand JP: Myocardial infarction redefined - a consensus document of The Joint European Society of Cardiology/American College of Cardiology Committee for the redefinition of myocardial infarction. J Am Coll Cardiol 2000, 36:959-969.

15. Oswald GA, Corcoran S, Yudkin JS: Prevalence and risks of hyperglycaemia and undiagnosed diabetes in patients with acute myocardial infarction. Lancet 1984, 1:1264-1267.

16. Malmberg K, Norhammar A, Wedel H, Ryden L: Glycometabolic state at admission: important risk marker of mortality in conventionally treated patients with diabetes mellitus and acute myocardial infarction: longterm results from the Diabetes and Insulin-Glucose Infusion in Acute Myocardial Infarction (DIGAMI) study. Circulation 1999, 99:2626-2632.

17. Oswald GA, Smith CC, Betteridge DJ, Yudkin JS: Determinants and importance of stress hyperglycaemia in non-diabetic patients with myocardial infarction. Br Med J (Clin Res Ed) 1986, 293:917-922.

18. Capes SE, Hunt D, Malmberg K, Gerstein HC: Stress hyperglycaemia and increased risk of death after myocardial infarction in patients with and without diabetes: a systematic overview. Lancet 2000, 355:773-778.

19. Marfella R, Verrazzo G, Acampora R, La Marca C, Giunta R, Lucarelli C, Paolisso G, Ceriello A, Giugliano D: Glutathione reverses systemic hemodynamic changes by acute hyperglycaemia in healthy subjects. Am J Physiol 1995, 268:E1167-E1173.

20. Kawano H, Motoyama T, Hirashima O, Hirai N, Miyao Y, Sakamoto T, Kugiyama K, Ogawa H, Yasue H: Hyperglycaemia rapidly suppresses flowmediated endothelium-dependent vasodilation of brachial artery. J Am Coll Cardiol 1999, 34:146-154

21. Ceriello A: Cardiovascular effects of acute hyperglycaemia: pathophysiological underpinnings. Diab Vasc Dis Res 2008, 5:260-268.

22. Mather AN, Crean A, Abidin N, Worthy G, Ball SG, Plein S, Greenwood JP: Relationship of dysglycaemia to acute myocardial infarct size and cardiovascular outcome as determined by cardiovascular magnetic resonance. J Cardiovasc Magn Reson 2010, 12:61.

23. Timmer JR, Ottervanger JP, de Boer MJ, Dambrink JH, Hoorntje JC, Gosselink AT, Suryapranata H, Zijlstra F, Hof AW Van 't: Zwolle Myocardial Infarction Study Group. Hyperglycaemia is an important predictor of impaired coronary flow before reperfusion therapy in ST-segment elevation myocardial infarction. J Am Coll Cardiol 2005, 45:999-1002.

24. Jensen CJ, Eberle HC, Nassenstein K, Schlosser T, Farazandeh M, Naber CK, Sabin GV, Bruder O: Impact of hyperglycaemia at admission in patients with acute ST-segment elevation myocardial infarction as assessed by contrast-enhanced MRI. Clin Res Cardiol 2011, 100:649-659.

25. Sinnaeve PR, Steg PG, Fox KA, Van de Werf F, Montalescot G, Granger CB, Knobel E, Anderson FA, Dabbous OH, Avezum A: GRACE Investigators. Association of elevated fasting glucose with increased short-term and 6month mortality in ST-segment elevation and non-ST-segment elevation acute coronary syndromes: the Global Registry of Acute Coronary Events. Arch Intern Med 2009, 169:402-409.

26. Su G, Mi S, Tao H, Li Z, Yang H, Zheng H, Zhou Y, Ma C: Association of glycemic variability and the presence and severity of coronary artery disease in patients with type 2 diabetes. Cardiovasc Diabetol 2011, 10:19.

27. Feinberg MS, Schwartz R, Tanne D, Fisman EZ, Hod H, Zahger D, Schwammethal E, Eldar M, Behar S, Tenenbaum A: Impact of the metabolic syndrome on the clinical outcomes of non-clinically diagnosed diabetic patients with acute coronary syndrome. Am J Cardiol 2007, 99:667-672.

28. Barsheshet A, Garty M, Grossman E, Sandach A, Lewis BS, Gottlieb S, Shotan A, Behar S, Caspi A, Schwartz R, Tenenbaum A, Leor J: Admission blood glucose level and mortality among hospitalized nondiabetic patients with heart failure. Arch Intern Med 2006, 166:1613-1619.

29. Kitada S, Otsuka Y, Kokubu N, Kasahara Y, Kataoka Y, Noguchi T, Goto Y, Kimura G, Nonogi H: Post-load hyperglycemia as an important predictor of long-term adverse cardiac events after acute myocardial infarction: a scientific study. Cardiovasc Diabetol 2010, 9:75.

30. Takada JY, Ramos RB, Avakian SD, Santos SM, Ramires JAF, Mansur AP. BNP and Admission Glucose as In-Hospital Mortality Predictors in Non-ST Elevation Myocardial Infarction. Scientific World Journal. 2012; 2012: 397915. Epub 2012 Jan 4.

31. Schroder J, Kahlke V, Staubach KH, Zabel P, Stuber F: Gender differences in human sepsis. Arch Surg 1998, 133:1200-1205

32. Oberholzer A, Keel M, Zellweger R, Steckholzer U, Trentz O, Ertel W: Incidence of septic complications and multiple organ failure in severely injured patients is sex specific. J Trauma 2000, 48:932-937.

33. Deshpande R, Khalili H, Pergolizzi RG, Michael SD, Chang MD: Estradiol down-regulates LPS-induced cytokine production and NFKB activation in murine macrophages. Am J Reprod Immunol 1997, 38:46-54.

34. Lazzeri C, Valente S, Chiostri M, Picariello C, Gensini GF. Acute glucose dysmetabolism in the early phase of ST-elevation myocardial infarction: the age response. Diab Vasc Dis Res. 2010; 7: 131-7. Epub 2010 Mar 4.

35. Valente S, Lazzeri C, Chiostri M, Giglioli C, Zucchini M, Grossi F, Gensini GF. Gender-related difference in ST-elevation myocardial infarction treated with primary angioplasty: a single-centre 6-year registry. Eur J Prev Cardiolog. 2012; 19: 233-40. Epub 2011 Mar 7.

36. Yu HT, Kim KJ, Bang WD, Oh CM, Jang JY, Cho SS, Kim JS, Ko YG, Choi D, Hong MK, Jang Y: Gender-based differences in the management and prognosis of acute coronary syndrome in Korea. Yonsei Med J 2011, 52:562-568.

37. Mansur A, De P, Favarato D, Avakian SD, Ramires JA: Trends in ischemic heart disease and stroke death ratios in Brazilian women and men. Clinics (Sao Paulo) 2010, 65:1143-1147.

doi:10.1186/1475-2840-11-47

Cite this article as: Takada et al.: In-hospital death in acute coronary syndrome was related to admission glucose in men but not in women. Cardiovascular Diabetology 2012 11:47

\section{Submit your next manuscript to BioMed Central and take full advantage of:}

- Convenient online submission

- Thorough peer review

- No space constraints or color figure charges

- Immediate publication on acceptance

- Inclusion in PubMed, CAS, Scopus and Google Scholar

- Research which is freely available for redistribution 